\title{
Chris Cannings: A Life in Games
}

\section{Timothy Bishop ${ }^{1} \cdot$ Mark Broom ${ }^{2} \cdot$ Richard Southwell ${ }^{3}$}

Published online: 21 December 2019

(C) The Author(s) 2019

\begin{abstract}
Chris Cannings was one of the pioneers of evolutionary game theory. His early work was inspired by the formulations of John Maynard Smith, Geoff Parker and Geoff Price; Chris recognized the need for a strong mathematical foundation both to validate stated results and to give a basis for extensions of the models. He was responsible for fundamental results on matrix games, as well as much of the theory of the important war of attrition game, patterns of evolutionarily stable strategies, multiplayer games and games on networks. In this paper we describe his work, key insights and their influence on research by others in this increasingly important field. Chris made substantial contributions to other areas such as population genetics and segregation analysis, but it was to games that he always returned. This review is written by three of his students from different stages of his career.
\end{abstract}

Keywords Evolutionary games · Evolutionarily stable strategy · War of attrition · Multiplayer games · Games on graphs

\section{Introduction}

Chris Cannings (1942-2017) is one of the most important contributors to the area of evolutionary games to date. While it is impossible to do justice to the breadth of his work in this arena, we attempt here to put in context some of his major observations. The three authors were all $\mathrm{PhD}$ students of Chris at the University of Sheffield from different periods of his career; Tim Bishop (1975-1977), Mark Broom (1990-1993) and Richard Southwell (20062009), and owe a great deal to him. We should point out that Chris was also a pioneer in

\footnotetext{
$凶 \quad$ Mark Broom

mark.broom@city.ac.uk

D. Timothy Bishop

d.t.bishop@leeds.ac.uk

Richard Southwell

richardsouthwell254@gmail.com

1 Leeds Institute of Data Analytics, University of Leeds, Leeds, UK

2 Department of Mathematics, City, University of London, Northampton Square, London EC1V 0HB, UK

3 Department of Mathematics, University of York, York YO10 5DD, UK
} 
mathematical genetics and leaves an equivalent body of work and impact on that field (which we touch on briefly in Sect. 3) and was interested in many aspects of the application of mathematics to understanding systems and their evolution; those who knew him will remember his enthusiasm when discovering a worthy problem. Much of his work in genetics as well as in game theory revolves around optimization, especially in evolutionary terms. This review, however, focuses on his evolutionary games work.

In the sections that follow we cover seven key themes of Chris' work. In Sect. 2 we discuss the war of attrition; in Sect. 3 matrix games and the Bishop-Cannings theorem; in Sect. 4 patterns of evolutionarily stable strategies; in Sect. 5 multiplayer games; in Sect. 6 models of some classic animal behaviours; in Sect. 7 games on static graphs and in Sect. 8 dynamic network models. Finally in Sect. 9 we discuss the overall impact of his work. First we discuss a little history, and how Chris started to work on evolutionary games.

In 1973, John Maynard Smith and Geoff Price described models of conflicts between members of the same species competing to take some limited resources. This work is generally considered the original article underpinning modern evolutionary games (although important earlier game-theoretic work exists on the sex-ratio game, e.g. see [70]). Their article, published in the journal Nature, described two-opponent symmetric, conflict models. These models were formulated to investigate optimal approaches to be taken by each opponent in response to the other's approach and to take into account the perceived value of winning the competition and taking the reward, be it food, habitat or partner. The motivation was the observation that intraspecific competition is often ritualistic and unlikely to cause harm; any challenge that led to physical injury could harm both winner and loser and make the participants more susceptible to attack and defeat by other local members of the same population or predators. Such observations had been interpreted as being beneficial to the species and community, but Maynard Smith and Price wished to explore if such behaviours could alternatively be interpreted as arising because they are beneficial to the individual. They postulated a number of distinct behaviours such as the "mouse" who always played the conventional low conflict approach, or "Hawk" who adopted an aggressive approach, while other behaviours responded to the approach of the opponent. In subsequent papers and discussions, the behaviour termed "mouse" was renamed as "Dove" but has the same character.

This elegant game theory model together with computer simulation suggested that the "limited war" (their term) solution could be advantageous to the individual in keeping with their speculation. In the discussion, they coined the phrase "Evolutionarily Stable Strategy" (ESS) to define the strategy which if adopted by the community would be advantageous over an interloper adopting a different strategy. In their terms, denoting $E[B, A]$ as the expected reward to an individual adopting strategy $\mathrm{B}$ in a competition against an individual adopting strategy $\mathrm{A}$, then if $\mathrm{A}$ is a strategy for which $E[A, A]>E[B, A]$ is true for every potential alternative strategy $\mathrm{B}$, then $\mathrm{A}$ is an ESS. Alternatively, if the inequality holds for some alternative strategies, but for other alternatives we have $E[A, A]=E[B, A]$, then $E[A, B]>E[B, B]$ was required for $\mathrm{A}$ to be an ESS (note that there is a more general condition for an ESS to hold involving the proportion $\epsilon$ of the population which play the alternative strategy, which reduces to the above for certain types of games, including those that were considered). The motivation for this formulation is that if a competitor adopts strategy A and the reminder of the population adopts A, then the population is stable to invasion by a mutant adopting the B strategy [108].

Chris was particularly intrigued by the potential to use game theory to explain behaviour and, when Tim Bishop arrived as a PhD student in 1975, proposed that their joint research should explore these models and the properties of the overall approach. 


\section{The War of Attrition}

John Maynard Smith (JMS) had proposed a contest (the "war of attrition") between two individuals in which the opponent willing to wait the longest wins. If a contest is ever to be settled, there must also be some disadvantages to the contestants in a long contest. If so, the only choice of strategy open to a contestant is of the period for which they are prepared to continue, and hence of the payoff they are prepared to accept. So, if one opponent's strategy is to wait for time $t_{1}$ and the opponent is willing to wait for $t_{2}$ (where $t_{1}>t_{2}$ ), then the contest ends at time $t_{2}$, with both contestants paying a cost $t_{2}$ and the winner gaining the reward of value $V$. For pure strategies $t_{1}$ and $t_{2}$ we get payoffs

$$
E\left[t_{1}, t_{2}\right]= \begin{cases}V-t_{2} & t_{1}>t_{2} \\ V / 2-t_{1} & t_{1}=t_{2} \\ -t_{1} & t_{1}<t_{2}\end{cases}
$$

JMS argued that no "pure" strategy (i.e. where a player always waits for the same amount of time $t$ ) could be the ESS, because the strategy of waiting just a moment longer would mean that this competitor would always win against the animal playing $t$, gaining the reward without incurring extra cost. The problem then is: How should a contestant choose a value of $t$, or, more precisely, is there a method of choosing $t$ which is an ESS? JMS argued that the optimal time would be an exponential time with mean $1 / V$, predominantly basing this on the exponential distribution and no information being conveyed about the contest ending in the next short interval. However, the argument was not complete and JMS mentioned this to Chris. JMS was not particularly worried about having a mathematically pure argument, indicating that he had no doubt about the solution. Chris took this as a challenge and set out first to provide the complete solution and then to generalize these models. The initial solution to the conflict as formulated by JMS, and confirming his conviction, was published in Bishop and Cannings [8].

\subsection{The Generalized War of Attrition}

In the "generalized war of attrition" [9], more general cost and reward functions are allowed than those proposed originally by JMS. For the generalized game with contest time $x$ having reward for winning $f(x)$ and cost of competing $g(x)$, the payoffs can be written

$$
E\left[t_{1}, t_{2}\right]= \begin{cases}f\left(t_{2}\right)-g\left(t_{2}\right) & t_{1}>t_{2}, \\ f\left(t_{1}\right) / 2-g\left(t_{1}\right) & t_{1}=t_{2}, \\ -g\left(t_{1}\right) & t_{1}<t_{2} .\end{cases}
$$

In such conflicts under reasonable assumptions about the cost and reward functions, there are either no ESSs or a unique solution, and in this latter case, the formal solution can be provided in closed form. For instance, if the strategy space involves competing without any time limit, the density function for the ESS is given by:

$$
p(x)=\frac{g^{\prime}(x)}{f(x)} \exp \left(-\int_{0}^{x} \frac{g^{\prime}(t)}{f(t)} \mathrm{d} t\right) \quad x \in[0, \infty) .
$$

Note that in the standard war of attrition, $f(x)=V$ (a constant) and $g(x)=x$.

Bishop et al. [11] expanded the conflict to the scenario where rewards varied randomly (the random rewards model) and also when the reward value differed between participants 
(e.g. when one was hungrier than the other) and/or the rewards progressed or escalated during the conflict; in these models, each opponent is aware of the reward for winning but unaware of the reward to the opponent (other than in distribution). If the reward takes on a series of discrete values, then the willingness to compete for longer increases, with each reward value corresponding to an interval time range which is non-overlapping with the time intervals corresponding to different rewards. One interesting difference with the standard war of attrition was that allowing different rewards for winning for different competitors means that the expected payoff is positive overall (in the war of attrition, the expected payoff is zero meaning that there is no difference in payoff (reward-cost) between strategies). In the random rewards model, the payoff is, on average, higher for the competitor with the highest value attached to the reward.

Bishop et al. [11] also provided a solution to the "graduated risks model" where competitors are willing to graduate the risk to which the conflict escalates, incurring an increased risk of injury (and cost). In this model, the solution depends on whether the cost of being injured exceeds the reward if successful. Of course, if the reward exceeds the cost of injury, then playing the most aggressive strategy is the ESS.

Bishop and Cannings [10] considered ordinal conflicts in which both the cost and the reward of competing were different between competitors. In these conflicts, the generalized war of attrition is further broadened so that both the reward and cost functions can differ between competitors. In these conflicts, the expected payoffs (when the ESS is played) depend only on the reward function and not on the specific form of the cost function.

\subsection{Further War of Attrition Models}

Haigh and Cannings [69] considered a multiplayer version of the war of attrition. Here individuals choose their waiting time as in the standard game; they considered four scenarios. In the first there was only a single reward, and individuals had to choose a waiting time at the start of the game. In the second, they could update their strategy every time that a player was observed to leave. The third and fourth variants were as for the first two except that there were multiple rewards, and a player received a reward based upon the order in which they left. In the first variant the ESS involved all players choosing a strategy with the following distribution function

$$
G(x)=(1-\exp (-x / V))^{1 /(m-1)} \quad x \in[0, \infty),
$$

where $m$ is the number of players. Subsequently, Helgesson and Wennberg [76] proved further results on this model.

Chris did further work on evolutionary game theory on Parker's model (Cannings [38], see also [124]), where two players choose amounts to pay, and the individual which pays the larger amount wins the reward, but each player also incurs a cost proportional to the amount they chose to pay. This is a variant on the war of attrition with the payoffs

$$
E\left[t_{1}, t_{2}\right]= \begin{cases}V-t_{1} & t_{1}>t_{2}, \\ V / 2-t_{1} & t_{1}=t_{2}, \\ -t_{1} & t_{1}<t_{2} .\end{cases}
$$

Chris considered the case where the strategy sets of the players are finite. In general there is no evolutionarily stable strategy in Parker's model. Using an interesting relationship, involving Dyck words (see e.g. [155]), Chris was able to determine the conditions for all strategies to be present in the stationary distribution of these systems. 


\section{Matrix Games and the Bishop-Cannings Theorem}

In considering the war of attrition, Chris realized that there were some general properties of ESSs which had become apparent. Bishop and Cannings [8] subsequently provided three theorems about ESSs. In terms of definitions, if, as above, a "pure" strategy involves playing for a fixed amount of time, a "mixed" strategy involves playing a random time determined by a defined probability distribution and the "support" of an ESS is the set of pure strategies which are included in the overall mixed strategy which is the ESS; Theorem 1 states that for each pure strategy in the support of the ESS, the payoffs for playing each such pure strategy against the ESS are the same. Theorem 2 states that if there are multiple ESSs for a particular conflict, then the support region for the first ESS cannot be a subset of the support region for the second ESS and vice versa. Below we define the concept of a matrix game and then state this theorem explicitly for this type of game.

A matrix game is one defined on pure strategies $\{1,2, \ldots, n\}$ where payoffs are given by a matrix $A=\left(a_{i j}\right)_{i, j=1, \ldots, n}$, where $a_{i j}$ is the payoff for playing strategy $i$ against strategy $j$. Mixed strategy payoffs are then given by

$$
E[\mathbf{p}, \mathbf{q}]=\mathbf{p} A \mathbf{q}^{\mathbf{T}} .
$$

For example, the classical Hawk-Dove game introduced by Maynard Smith and Price [109] is a matrix game with two strategies Hawk $(H)$ and Dove $(D)$ and payoffs $E[H, H]=$ $(V-C) / 2, E[H, D]=V, E[D, D]=V / 2$ and $E[D, H]=0$ (individuals compete for a reward $V$, Hawks beat Doves, but losers in Hawk versus Hawk contests pay a cost $C$ ).

The support of $\mathbf{p}$ is $S(\mathbf{p})=\left\{i: p_{i}>0\right\}$. Defining $T(\mathbf{p})$ to be the set pure strategies which have equal payoffs against the strategy $\mathbf{p}$, i.e.

$$
T(\mathbf{p})=\{i: E[i, \mathbf{p}]=E[\mathbf{p}, \mathbf{p}]\},
$$

for matrix games Theorem 2 states:

If $\mathbf{p}$ is an ESS of the matrix game $A$ and $\mathbf{q} \neq \mathbf{p}$ is such that $S(\mathbf{q}) \subseteq T(\mathbf{p})$, then $\mathbf{q}$ is not an ESS of matrix game $A$ (which is a slightly stronger result than that stated above).

Theorem 2 is now widely known as the Bishop-Cannings theorem and is a fundamental result in the theory of ESSs. In particular, we see its use in studying patterns of ESSs in Sect. 4. On the basis of this result, it follows (Theorem 3) that if an ESS involves playing each pure strategy with some probabilities, then that ESS is unique. We have stated the above results for a set of discrete pure strategies, but it holds for a continuous set too, such as for the war of attrition as mentioned above.

Finally, Bishop and Cannings [8,9] developed an algorithmic approach to evaluate potential ESS solutions for any competition whether continuous or discrete. A specific algorithm for finding ESSs of matrix games was given by Haigh [66] (but see Abakuks [1], who showed that this does not always hold for non-generic cases). Depending on the payoff matrix, games can have no, one or several ESSs, an observation first made by John Haigh. Chris and Glenn Vickers subsequently worked extensively on the issue of identifying the characteristics of the payoff matrices leading to games with specific combinations of ESS supports (see Sect. 4).

Bomze [12] returned to the issue of identifying ESSs in matrix games and gave a detailed discussion of an algorithm to enumerate and verify candidate ESS solutions. In Sect. 4 we discuss further recent developments relating to patterns of ESSs which build on this work, especially in Bomze et al. [15].

We should note that the definition of an ESS as given above allows for resistance to invasion to require an ever smaller fraction of the population, denoted by $\epsilon$ as mentioned in 
Sect. 1, for successive invading strategies, and so there may be no minimum fraction that works for all invading strategies. This was pointed out by Vickers and Cannings [161]. If there is such a fraction, then an ESS is said to have a uniform invasion barrier. It was shown by Hofbauer et al. [79] that such a uniform invasion barrier exists for matrix games. There are in fact many notions of evolutionary stability with a range of different properties that we shall not discuss in detail here. For a snapshot of those defined by the time of their article's publication, see Apaloo et al. [3]; see also Lessard [98].

More recently, a number of important lines of research have followed building on the mathematical framework developed primarily by Chris; while there are many citations of this early work, we have chosen a few of the more notable strands (which are not covered elsewhere in this paper) to illustrate the breadth of activity.

Eshel [54] considered a situation with a continuous strategy set, related to the war of attrition. In particular he explored the relationship between the ESS and continuous stability, that is if the population adopts a strategy similar to but not identical to the ESS, there is an advantage of adopting a strategy closer to the ESS, see also Eshel and Sansone [55]. He showed necessary and sufficient conditions for the ESS to be continuously stable. This was a forerunner of the ideas of adaptive dynamics (see [60]), which we discuss briefly in Sect. 6.

Vincent and Brown [165] developed an approach to modelling evolution which explicitly incorporates ecological factors using their G-function methodology. This was developed following a series of earlier works such as Vincent and Brown [164] and Brown and Vincent [34]. Vincent and Brown [166] contrasted ESSs and Nash equilibria and the importance of stability concepts in evolutionary models. They developed these ideas more together with Apaloo in Apaloo et al. [3], where important concepts of evolutionary stability were discussed (see also [2]) and contrasted.

Rapoport [138] discussed in detail the relationship between ESSs and conventional gametheoretic approaches stressing the equivalence of studying behaviour in biological systems with traditional game methodology. We note that while there is a strong theoretical link between conventional games, in particular based on economic ideas, and evolutionary games based on biology, the two areas have for the most part developed in parallel without much interaction. This has changed more recently, e.g. see Sandholm [143].

\subsection{Asymmetric Games}

One of the key developments that emerged early in the theory of evolutionary games is that of asymmetric games. While Chris did not work on this explicitly, this important work built on his contributions.

The war of attrition and similar models assumed that while there could be differences in the value of the reward for winning, there was no asymmetry in terms of external information prior to or during the contest. However, there are many conflicts which have asymmetries, such as when one of the participants is the current owner of a territory, so that each conflict is between an owner and an intruder. In such scenarios, the nature of the ESS differs with this external information, the asymmetry, assisting in defining the optimal solutions. In such games, there are often two solutions [124], one regarded as common sense in which the owner is willing to remain for longer and an intruder investigates whether the territory can be won with limited expenditure or the inverse solution where the owner retreats when challenged. Hammerstein [71] showed that even a modest modification of the Hawk-Dove game could result in games with two ESS solutions. These two strategies are ESSs but not unexpectedly in the owner-intruder example; the former is the commonest scenario in nature. However, 
there are real-world examples of the latter (see Parker [124] for examples). It was shown in Selten [145] that for a wide class of such asymmetric games, it was not possible to have mixed ESSs, so that the only ESSs are pure.

Hammerstein and Parker [72] examined the conditions under which multiple ESS solutions might be found. They considered the issue of incomplete information (that is imprecision in for instance the owner-intruder conflict) or scenarios under which fighting approaches were not continuous, e.g. Hawk vs Dove discrete behaviours as compared to the continuous nature of the time choice in the war of attrition. They found that the criteria which allowed such counter-intuitive solutions to be feasible included that the information had to be predominantly correct and that the decision to remain had to be taken early in the conflict with no early opportunity for avoiding the impact of a decision to compete and that behaviours were continuous.

The theory of asymmetric games, in particular in relation to owner-intruder relationships, has been developed in a number of directions. A significant series of papers was by MestertonGibbons, Sherratt and co-workers, who have considered various extensions to the theme, for example in Mesterton-Gibbons et al. [113,114]. In particular, the study by Gibbons and Sherratt [112] considers the concept of infinite regress previously raised by JMS, where if an owner is displaced by an intruder in an owner-intruder game the roles are then reversed, so that the second ESS described above might lead to an infinite contest. A review of this and other work is presented in Sherratt and Mesterton-Gibbons [146].

\subsection{Genetic Models and Discrete Dynamics}

While JMS coined the term evolutionarily stable strategy, the implicit assumption was that when the whole population adopted that strategy, such a population could not be invaded by an interloper or a mutation which translated into a different strategy. However, it is not clear that the population dynamics would translate into that ESS being achieved within the population if it did not start with all individuals adopting that strategy. There is a huge literature on evolutionary dynamics considering this and other questions, including a range of different dynamics, and we shall not discuss it here. In particular the most widely used dynamics is the replicator dynamics developed in Taylor and Jonker [158], where the population evolves in continuous time using differential equations. We note that ESSs and stable rest points of the dynamics are not identical (see [78]). While this, and other, continuous-time dynamics are more widely used in the literature, given his interest in genetic models, Chris was more interested in discrete-time dynamics, and a discrete version of the replicator equation appears in Bishop and Cannings [9].

In this discrete-time model, the assumption is made that the fitness of an individual is related to the strategy adopted plus a marginal fitness attributable to all other activities which is constant among community members. The dynamics can then be examined using approaches such as that of Kimura [86] and Kingman [87]. The interests of these investigators were the evolution of allele frequencies; in such a scenario, the payoff matrix $A$ is symmetric (in the genetics example, this represents the fitness of the heterozygote for off-diagonal elements) and it is known that the population evolves to a stable equilibrium if one exists. The dynamic model considers that the representation of a particular type in the population in generation $G_{n}$ is proportional to the frequency in generation $G_{n-1}$ multiplied by the fitness of that type compared to the rest of the population. In fact, in this scenario of allele frequency changes, the mean viability increases monotonically from generation to generation towards the asymptotic equilibrium. 
Chris had employed such an approach successfully when examining the effect of selection on a multiallelic system when the population was split between multiple niches and mating happened within each niche [36]. Assuming the population is large, Bishop and Cannings [9] showed that for the generalized war of attrition, if there is an ESS, then this strategy is both attainable in a large population and achieved asymptotically. However, in the general setting the mean fitness of the population does not necessarily monotonically increase from generation to generation. Chris was particularly interested in trying to understand the relationship between the ESS and dynamic stability especially in the scenario where the strategy adopted in conflicts was genetically determined. However, it is fair to say that limited progress was made on the overall problem and it remains an interesting, unresolved issue.

One outstanding problem is to consider how the imposition of germline inheritance on conflict behaviour might evolve, as opposed to the phenotypic modelling which had been discussed previously. Hines and Bishop [77] and Cressman and Hines [47] showed that while there were situations in which the ESS was not locally stable, restricting the mode of inheritance by excluding situations which were less biologically plausible such as overdominance, meant that the ESS was locally stable. Some recent works have been carried out on such genetic models in Fishman [56,57], where a methodology of how to approach these problems was introduced and some analyses for classical games including the Hawk-Dove game and Prisoner's dilemma are carried out.

\section{Patterns of ESSs}

We have seen above how the Bishop-Cannings theorem means that certain combinations of ESSs are not possible. What other results can be found that tell us more about what is possible? This was the question investigated by Chris with Glenn Vickers and later by coworkers including Mark Broom in a series of papers from the late 1980s. It is not feasible to consider all of the possible ESS vectors that can occur for a given matrix so, as in the Bishop-Cannings theorem, they concentrated on the supports of the ESSs. The idea of a pattern of ESSs was introduced in Vickers and Cannings [163]. The pattern of the matrix game $A$ is the collection of supports of the ESSs of $A$, i.e. if $\mathbf{p}_{1}, \mathbf{p}_{2}, \ldots, \mathbf{p}_{N}$ is the list of all ESSs of $A$, then

$$
\mathcal{P}(A)=\left\{S\left(\mathbf{p}_{1}\right), S\left(\mathbf{p}_{2}\right) \ldots, S\left(\mathbf{p}_{N}\right)\right\} .
$$

A collection of subsets of $\{1,2, \ldots, n\}, \mathcal{P}$, is an attainable pattern if there is some matrix $A$ for which Eq. (8) holds. It is attainable on $n$ pure strategies if there is such a matrix which is $n \times n$. We note that since the numbers here are arbitrary, any re-numbering of an (un)attainable pattern also yields an (un)attainable pattern. Cannings and Vickers sought to find which patterns of ESSs are possible, by generating new exclusion results, as well as general construction methods for attainable patterns.

A pattern $\mathcal{P}$ on $n$ pure strategies is maximal if it is attainable, and $\mathcal{P} \cup S_{1} \cup \ldots \cup S_{k}$ is not attainable for any $k$ and non-empty $S_{1}, \ldots, S_{k} \subset\{1,2, \ldots, n\}$. The following conjecture, if true, would mean that consideration of only maximal patterns is sufficient.

Conjecture If $\mathcal{P}$ is attainable on $n$ pure strategies, then $\mathcal{P} * \subset \mathcal{P}$ is also attainable on $n$ pure strategies [42].

A weaker result, that if $\mathcal{P}$ is attainable on $n$ pure strategies, then $\mathcal{P} * \subset \mathcal{P}$ is attainable on $n+k$ pure strategies for $k \geq K$ for some $K$, was shown in Broom [17]. 
The full set of attainable patterns for the cases $n=2,3,4$ and the set of maximal patterns (subject to two unknown cases) for $n=5$ is shown in Cannings and Vickers [42,43] (see also [162]). For instance, the full set of attainable patterns for $n=3$ and $n=4$ is given by $n=3:\{(1,2,3)\},\{(1,2),(1,3)\},\{(1,2),(3)\},\{(1),(2),(3)\}$ $n=4:\{(1,2,3,4)\},\{(1,2,3),(1,2,4)\},\{(1,2,3),(2,4),(3,4)\},\{(1,2,3),(4)\}$, $\{(1,2),(1,3),(1,4)\},\{(1,2),(1,3),(4)\},\{(1,2),(2,3),(3,4),(1,4)\}$, $\{(1,2),(3),(4)\},\{(1),(2),(3),(4)\}$.

The case of symmetric games with payoffs $a_{i j}=a_{j i}$, which can be used in genetics with strategies corresponding to alleles, was considered in Cannings et al. [44], as discussed in Sect. 3 .

\subsection{Exclusion Rules}

There are a number of exclusion rules, most of which appear in Vickers and Cannings [163]. We show the two simplest rules below. The first of these is the most useful and is in fact just an application of the Bishop-Cannings theorem.

Exclusion rule 1 Anti-chain. If $S_{1} \subseteq S_{2}$, then no pattern can contain both $S_{1}$ and $S_{2}$.

Exclusion rule 2 No triangles. If $Q \subseteq\{4,5, \ldots, n\}, S_{1}=\{1,2\} \cup Q, S_{2}=\{1,3\} \cup Q$ and $S_{3}=\{2,3\} \cup Q$, then no pattern can contain $\left\{S_{1}, S_{2}, S_{3}\right\}$.

All non-obtainable patterns for the case $n=3$ above can be excluded using these two rules only (the second rule excludes the triangle $\{(1,2),(1,3),(2,3)\})$. For $n=4$ and above we need these two rules, but also others.

\subsection{Constructing Attainable Patterns}

There are also a number of construction methods, see Broom et al. [24] (also [26]) and more recently Bomze et al. [15]. The simplest of these from Cannings and Vickers [41] involves the idea of a clique matrix. An $n \times n$ matrix $A$ is a clique matrix if $a_{i i}=0$ for all $i$ and $a_{i j}=a_{j i}= \pm 1$ for all $i \neq j$. A set of pure strategies $S$ forms a clique if and only if $a_{i j}=1 \forall i, j \in S$ with $i \neq j$ and there is no $k \notin S$ s.t. $a_{k j}=1 \forall j \in S$.

It is easy to show that there is an ESS of $A$ with support $S$ if and only if $S$ is a clique, and so $\mathcal{P}(A)$ is simply the set of cliques of $A$. All attainable patterns for $n=3$ can be found using cliques, but for larger $n$ we need other construction methods, and cliques fail to find an increasing proportion of them.

Recent work considering a variant of the clique matrix defined above, where $a_{i j}=a_{j i}=1$ or 0 is given in Wang et al. [168] (see also [74]). Here the precise network was chosen for application motivational reasons, and the payoff matrix corresponds to a graph adjacency matrix where $1(0)$ represents the presence (absence) of a social link, although we note that this matrix class leads to more complex conditions and thus is less useful for constructing ESS patterns.

\subsection{The Maximum Number of ESSs}

The maximum number of ESSs possible for $n \times n$ matrices was denoted in Broom et al. [23] by $M_{n}$. In particular they proved that $M_{n} M_{m} \leq M_{n+m}$ and that

$$
\lim _{n \rightarrow \infty}\left(M_{n}\right)^{1 / n}=\gamma,
$$


for some $\gamma \leq 2$.

A lower bound for $\gamma$ of $30^{1 / 9} \approx 1.459$ was found in Broom et al. [23]. In fact a value almost as good, $3^{1 / 3} \approx 1.442$, can be found using clique matrices. This bound was recently improved on in Bomze et al. [15] to $15120^{1 / 24} \approx 1.493$. In that paper the authors build on earlier work Bomze [12,13] to develop ways to tackle this and related problems (as in Broom et al. [23] the focus was on the standard quadratic optimization problem which has a number of other applications too). They found lower bounds for the maximum number of ESSs for all $n \leq 24$ and also investigated methods for looking for ESSs for larger $n$ more generally. This work was developed further in Bomze and Schachinger [14] in the same issue of dynamic games and applications as this review, and this paper contains a number of interesting analyses.

Upper and lower bounds on the number of ESSs of a given mean support size

$$
f(\alpha)=\lim _{n \rightarrow \infty}\left(s_{\alpha n}\right)^{1 / n},
$$

where $s_{n}(x)$ is the maximum number of ESSs of mean support size $x$ on an $n \times n$ matrix, were found in Broom [16]. Here it was shown that $f(\alpha)$ is continuous on the rational numbers.

The above bounds for $\gamma$ and $f(\alpha)$ are still very wide, and it will be a considerable challenge to improve them. Nevertheless, the recent work in Bomze et al. [15] and Bomze and Schachinger [14] is the first significant improvement for many years and hopefully there is more of such improvements to come.

\subsection{The Expected Number of ESSs}

In practice we are unlikely to have a very large number of ESSs of a matrix game, and the question of how many ESSs we would expect to see was considered in Cannings and Vickers [41], when considering randomly generated clique matrices. The same problem for more general matrices was considered in Haigh $[67,68]$. We note that here the distribution of the payoffs affects the answer; in Haigh [67] all payoffs were assumed to be i.i.d from a uniform distribution. Large ESSs supports were shown to be very unlikely to occur in Haigh [68].

This problem is significantly complicated by the stability condition required to make a Nash equilibrium into an ESS, and addressing the problem of the number of Nash equilibria that can occur is an easier problem to tackle, as in Han et al. [73] and Duong and Han [50], which both actually considered multiplayer games (as defined in Sect. 5) as well as two-player games. As well as proving general results Duong and Han [50] showed that, under specific distributional and independence assumptions, the probability of an internal equilibrium is simply $1 / 2^{n-1}$. This work is part of a series of papers in this area, see Duong and Tran [51] and Duong et al. [52].

Due to its general mathematical formulation on quadratic forms, the study by Broom et al. [23] has been cited in various later mathematical works with no clear biological or game-theoretic connection. A relevant biological connection is in the work of Leinster and Meckes [95]. Here the authors develop a model of biological diversity and use a similarity matrix between the species (values being between 0 and 1, where the similarity of a species to itself is 1). They are interested in maximizing diversity and use a number of entropy measures. Interestingly, the conditions for optimizing entropy closely resemble those for ESSs of symmetric evolutionary games, when the dissimilarity matrix (1 minus the similarity matrix) is used (see e.g. Cannings et al. [44] where such symmetric matrices are considered). 


\section{Multiplayer Games}

Following on from the above work, together again with Broom and Vickers, Chris considered general multiplayer evolutionary games. He had previously published one paper on multiplayer games [69], which is mentioned in Sect. 2. This began with work on an extension of the theory of matrix games to the general situation of multiplayer games in Broom et al. [28].

\subsection{Multiplayer Matrix Games}

Broom et al. [28] considered the situation of an infinite population, where randomly selected groups of size $m$ play a game where there are $n$ pure strategies available. In the most general case the specific ordering of all players could be important, and there would be a distinct set of payoffs, one to each of the $m$ players, for each ordered strategy selection $i_{1} i_{2} \ldots i_{m}$. However, they concentrated on symmetric games, where the payoff to any given individual depended only upon its own strategy and the combination of the strategies of the other players, irrespective of their ordering.

Thus, the payoff to an individual playing $i_{1}$ against players playing $i_{2}, \ldots, i_{m}$ satisfies $a_{i_{1} i_{2} \ldots i_{m}}=a_{i_{1} \sigma\left(i_{2}\right) \ldots \sigma\left(i_{m}\right)}$ for any permutation $\sigma$ of the indices $i_{2}, \ldots, i_{m}$. Matrix games are just a special case of this situation with $m=2$.

They showed that the Bishop-Cannings theorem fails (i.e. it is possible to have two ESSs where the support of one is a subset of the support of the other) for $m \geq 3$, that it is not possible to have two ESSs with the same support for $m=3$, but that this is possible for $m \geq 4$. They considered in some detail the case with $n=2$, where they fully categorized the possible sets of ESSs (not just their supports) that could occur for a single game in the generic case; this case was further developed in Pena et al. [131]. In this case the payoffs can be simply denoted $\alpha_{i j}$ where $i=1,2$ represents the player's strategy, and $j=0, \ldots, m-1$ represents the number of its opponents that play strategy 1 , the remaining $m-1-j$ thus playing strategy 2. Broom et al. [28] showed that for generic games, mixed ESSs are represented by the zeros of the following polynomial where the derivative is less than 0 ,

$$
h(p)=\sum_{l=0}^{m-1}\left(\begin{array}{c}
m-1 \\
l
\end{array}\right) \beta_{l} p^{l}(1-p)^{m-l-1},
$$

where $\beta_{l}=\alpha_{1 l}-\alpha_{2 l}$. Pure strategy 1 (2) is an ESS if $\beta_{m-1}>0\left(\beta_{0}<0\right)$.

This work was developed further in Bukowski and Miękisz [35] who proved extensions of classical results for matrix games, such as that a strategy $\mathbf{p}$ is an ESS if and only if it is locally superior. They also gave the nice example below to illustrate that a game can have more than one ESS with the same support for $m=4$.

Example 1 ([35]) Consider the game with payoffs $\alpha_{11}=\alpha_{22}=-\frac{13}{96}$, $\alpha_{13}=\alpha_{20}=-\frac{3}{32}$ and $\alpha_{10}=\alpha_{12}=\alpha_{21}=\alpha_{23}=0$. Thus $\beta_{0}=3 / 32$, $\beta_{1}=-13 / 96, \beta_{2}=13 / 96, \beta_{3}=-3 / 32$ giving

$$
\begin{aligned}
h(p) & =-\frac{3}{32} p^{3}+\frac{13}{32} p^{2}(1-p)-\frac{13}{32} p(1-p)^{2}+\frac{3}{32}(1-p)^{3} \\
& =-\left(p-\frac{1}{4}\right)\left(p-\frac{1}{2}\right)\left(p-\frac{3}{4}\right),
\end{aligned}
$$

and thus the game has two internal ESSs at $\mathbf{p}=(1 / 4,3 / 4)$ and $\mathbf{p}=(3 / 4,1 / 4)$ (and no pure ESSs). 
Broom et al. [28] also considered the case with $n=m=3$, the simplest case where both $m$ and $n$ are greater than two, for the special case of supersymmetric games, where $a_{i_{1} i_{2} \ldots i_{m}}=a_{\sigma\left(i_{1}\right) \sigma\left(i_{2}\right) \ldots \sigma\left(i_{m}\right)}$ for any permutation $\sigma$ of the indices $i_{1} i_{2}, \ldots, i_{m}$, which is the extension to multiple players of the genetics-based model of Cannings et al. [44]. The range of combinations of ESSs that resulted showed the difficulty in dealing with this problem more generally.

Platkowski has written a number of papers on multiplayer evolutionary games $[134,136$, 137]. Recently he introduced the idea of evolutionary coalitional games [135], i.e. incorporating classical coalition games into the evolutionary setting, where the population evolves following the replicator dynamics. Here he showed that, contrary to the corresponding evolutionary models in well-mixed populations, cooperation can be supported in the system in the long run. The two-strategy game of Broom et al. [28] was also recently used in laboratory experiments to investigate human cooperative behaviour in Kuzmics and Rodenburger [92].

The stochastic stability for a finite population playing three-player games with two strategies was the subject of a series of papers by Miekisz and co-authors, where they studied this situation for the well-mixed case [85], a spatially structured population [115] and a population where there was an added time delay in the games [116] (see also Miekisz et al. [117] in the same journal issue as this review).

This area was further explored more recently by Gokhale and Traulsen [62,63], Wu et al. [170], see also Han et al. [73] and Duong and Han [50], as discussed in Sect. 4.4. The study by Gokhale and Traulsen [64] is a good review of work up until 2014. In Gokhale and Traulsen [62], the authors investigated both infinite and finite populations. The question of the number of Nash equilibria (as opposed to ESSs) it was possible to have in a multiplayer game was considered. In general it was shown that the maximum number of internal equilibria possible is $(m-$ $1)^{n-1}$.

The possible emergence of multiplayer games from pairwise ones was considered in $\mathrm{Wu}$ et al. [171]. Here the authors considered pairwise games that occurred in sets that broke up at rates that depended upon their composition, and which led to games that were multiplayer in character, with payoff functions as in multiplayer matrix games. These were compared to linear independent combinations of pairwise games to show how multiplayer games might emerge. The effect of a multiplayer game being the result of non-independent pairwise ones is also the subject of Sect. 5.2.

There are also more computational applications for evolutionary games and in particular multiplayer evolutionary games. In Rota Bulo and Pelillo [142] (see also [127]) evolutionary game methodology was applied to the problem of hypergraph clustering, that is the process of finding the most coherent groupings from a set of objects using high-order (i.e. not just pairwise) similarities. Here the authors devised a hypergraph clustering game and showed equivalence between the ESSs of the game (with multiplayer ESSs of level 1 as defined in [28]) and dominant set clusters, an important concept from clustering theory.

The concept of games, including evolutionary games, has been combined with quantum dynamics to give quantum games, [53] with potential applications to evolution at the molecular level. Evolutionary aspects have particularly been considered by Iqbal and colleagues for the two-player and multiplayer settings [45,80-82]. See Orlin Grabbe [122] for an introduction to this area. 


\subsection{Knockout Contests and Swiss Tournaments}

In Broom et al. [30,31] the same authors considered pairwise games built into a dependent structure that meant, unlike for standard matrix games, players did not play games independently of their strategy and so a more complex multiplayer contest resulted. The game was built on knockout contests like the Wimbledon tennis tournament (although this involves some non-random element by seeding the leading players) where individuals are randomly paired, but only the "winner" qualifies for the next round, and a similar random draw is carried out between the winning players. This process starts with $2^{n}$ players and is repeated until a final between two players occurs. An individual receives a reward $V_{j}$ for being eliminated in the round of $2^{j}$ players, with the winner receiving $V_{0}$. Individuals can also receive costs from a contest, depending upon their strategic choice and that of their opponent. The probability of progression also depends upon these choices, and this means that later pairings are depended upon strategy. They considered two versions of the game, where individuals had to play the same pure strategy in every round (the fixed case) and where they could change it, following a (possibly round-dependent) mixed strategy, every round.

An example component game considered was the Hawk-Dove game. Here Hawk versus Hawk contests resulted in a cost of $C$, and otherwise there were no costs, as standard. Here the reward $V$ was replaced by progression to the next round. Thus Hawks always beat Doves, and contests between individuals playing the same strategy resulted in each progressing with a probability of $1 / 2$. For the fixed strategy case there are fewer Hawks in the population the more rounds there are, as Hawks are more likely to progress to later rounds and have contests against increasingly Hawkish opponents; the distribution of observed contests can also be significantly different from the comparable case with independent contests.

This game was further developed by Broom and Cannings [18] to consider a different type of contest where all players were involved in every round, based upon the "Swiss tournament" common in chess competitions. Individuals should be aggressive in early rounds and, if successful, later rounds. Losers however should become less aggressive, and this nicely mirrors the "loser effect" described in the models below.

Classic models of dominance hierarchy formation consider a series of contests within a group where winning increases the chance of winning another contest (winner effects) and/or losing decreases this chance (loser effects). Various models have considered the evolution of dominance hierarchies under winner and loser effects and in particular whether and how fast a linear hierarchy can be established including Dugatkin [48], Dugatkin and Dugatkin [49], Mesterton-Gibbons and Dugatkin [111] and Kura et al. [89]. In Dugatkin [48] individuals could assess the relative fighting ability of their opponent and concede if it was above a certain threshold. Kura et al. [90] considered an explicitly game-theoretic model where individuals could select their concession threshold strategically. Here a pure strategy solution was found, and this corresponded with fast establishment of a linear hierarchy.

Through a detailed study of data in hens and cichlid fish, Lindquist and Chase [103] (see also [46,102]) found a dynamic relationship more complex than the linear dominance hierarchies from previous models. They suggested that the outcomes of hierarchies to a great extent depended upon two rules only. Rule 1 was that there were aggressive contests between all pairs of animals, and rule 2 that intransitive configurations of attacks were transformed to transitive ones faster than vice versa, but that there is a continuous dynamic updating.

A model of a population of birds arriving at a nesting area was developed in a further series of papers by Chris and colleagues. Here birds arrived sequentially and had to select one of the nests sites, which varied in quality. Good sites were likely to be contested by later arrivals, so sometimes it was best to select intermediate sites to avoid costly contests. They 
thus arrived at a set of dependent pairwise contests which combined to be a potentially very complex multiplayer game. Two extremes of birds with great and poor calculating powers were considered in Broom et al. [27,29], respectively. An interesting situation where birds cooperated by selecting the worst sites and avoiding all conflicts, reminiscent of Tit-for-Tat in the Iterated Prisoner's Dilemma, occurred in Broom et al. [25].

\subsection{Multiplayer Games in Structured Populations}

We shall discuss structured populations in more detail in Sects. 7 and 8. However, it is worth discussing them a little here in connection to multiplayer games. While none of the work below in this section was done by Chris, both structured populations and multiplayer games were a significant interest of his and his ideas influenced some of the work below. The classical game models from previous sections have all relied on the population being unstructured, or well-mixed, with every pair or group of individuals as equally likely to interact as any other of the same size. Populations with structure, based upon an underlying graph representing possible interactions (evolutionary graph theory), were introduced in Lieberman et al. [101]. The early models did not involve games at all, and when games were introduced [119,121] inevitably these were pairwise, reflecting the edges of the graph. Multiplayer games were introduced on a graph by Santos et al. [144] (see also [99,100]) where each individual played a multiplayer game involving all of its neighbours (and so if it had $k$ neighbours it would also be involved in $k$ further games centred upon each of them).

For finite structured populations, it is known that when considering the two-player Prisoner's dilemma, an important property to assess whether cooperation evolves is the structure coefficient. How does this extend to multiplayer games? This question was addressed by McAvoy and Hauert [110] and Pena et al. [132] who considered the specific coefficients to be used; a more general type of structural coefficient is needed here, and typically $m-1$ are needed for an $m$ player game (see [141]). This has only been developed for specific types of graph so far. Pena et al. [133] consider the concepts of volume order and containment order which can use structure coefficients to assess the relative likelihood of the evolution of cooperation for different structures in a wide class of games.

Multiplayer games, including those with variable size, were introduced with the development of a new modelling framework in Broom and Rychtár [22] (see also [32,125,126]). This is a flexible framework with many possibilities, and a range of structures, games and dynamics has been considered. See also Tarnita et al. [157] and Pena and Rochat [130] for alternative ideas. Having variability in group size can have significant effects on the evolution of populations, see Pena and Noldeke [128,129] and Broom et al. [33]. For instance Pena and Noldeke [128] found conditions on the payoffs for when increased variability would favour, or inhibit, the evolution of cooperation for a specific class of games.

\section{Models of Classical Animal Behaviour}

The early classical evolutionary game theory models, such as the sex-ratio game, HawkDove game or war of attrition, were developed with a specific biological application in mind, but were nevertheless very simple idealized games designed to show key insights with clarity. Subsequently models of animal behaviour which incorporated more realistic detail were developed, and now there are very many such models. Chris and colleagues developed some of these models in the 1990s and early 2000s. We shall consider three types of model 
here: the co-evolution of predator-prey interactions, parasitoid superparasitism and sperm competition models. In each case we shall briefly describe the mathematical model, before discussing the key conclusions and connections to later work.

\subsection{Predator-Prey Co-evolution}

Models of predator-prey interactions are some of the earliest in mathematical biology $[105,167]$. These early models were not game-theoretic, but there have been a number of models developed from this classical interaction that have involved strategic behaviour of the predators and/or the prey. An influential model of this type was developed by Marrow et al. [107] (see also [106]) which considered the classic predator-prey population dynamic model, but where there was also a slower evolutionary process affecting the body sizes of both predators and prey.

They considered a polymorphic population with two types of prey, with slightly different body sizes $s_{1}$ and $s_{1}+\epsilon_{1}$, and two types of predator, with body sizes $s_{2}$ and $s_{2}+\epsilon_{2}$. The population then followed the ecological dynamics below:

$$
\begin{aligned}
\frac{\mathrm{d} x_{1}}{\mathrm{~d} t} & =x_{1}\left(r_{1}+\alpha_{s_{1}}\left(x_{1}+x_{1}^{\epsilon}\right)+\beta_{s_{1}, s_{2}} x_{2}+\beta_{s_{1}, s_{2}+\epsilon_{2}} x_{2}^{\epsilon}\right), \\
\frac{\mathrm{d} x_{1}^{\epsilon}}{\mathrm{d} t} & =x_{1}^{\epsilon}\left(r_{1}+\alpha_{s_{1}+\epsilon_{1}}\left(x_{1}+x_{1}^{\epsilon}\right)+\beta_{s_{1}+\epsilon_{1}, s_{2}} x_{2}+\beta_{s_{1}+\epsilon_{1}, s_{2}+\epsilon_{2}} x_{2}^{\epsilon}\right), \\
\frac{\mathrm{d} x_{2}}{\mathrm{~d} t} & =x_{2}\left(r_{2}+\gamma_{s_{1}, s_{2}} x_{1}+\gamma_{s_{1}+\epsilon_{1}, s_{2}} x_{1}^{\epsilon}\right), \\
\frac{\mathrm{d} x_{2}^{\epsilon}}{\mathrm{d} t} & =x_{2}^{\epsilon}\left(r_{2}+\gamma_{s_{1}, s_{2}+\epsilon_{2}} x_{1}+\gamma_{s_{1}+\epsilon_{1}, s_{2}+\epsilon_{2}} x_{1}^{\epsilon}\right),
\end{aligned}
$$

where $x_{1}\left(x_{1}^{\epsilon}\right)$ is the population size of the prey with body size $s_{1}\left(s_{1}+\epsilon_{1}\right)$, and $x_{2}\left(x_{2}^{\epsilon}\right)$ is the population size of the predator with body size $s_{2}\left(s_{2}+\epsilon_{2}\right)$. The $r$ parameters (both positive) represent low density growth, the $\alpha$ s (negative) are self-limiting parameters for the prey, and the $\beta$ s (negative) and $\gamma$ s (positive) are predator-prey interaction terms, where any given predator type is more likely to kill certain types of prey than others.

The result of these ecological dynamics then determined whether the mutant body sizes $s_{1}+\epsilon_{1}, s_{2}+\epsilon_{2}$ could invade a population $\left(s_{1}, s_{2}\right)$ (we will omit discussions of the conditions here). Thus over time the body sizes within the population would evolve, governed by the predator-prey interactions.

For plausible functional forms for these parameters, Marrow et al. [107] identified ten distinct evolutionary scenarios, including a continuous "red-queen" cycling though the body sizes. This paper demonstrates some of the possibilities of trait evolution and was cited in some of the important early works in the theory of adaptive dynamics (such as $[60,88,93]$ ), which is a very flexible and general mathematical theory of trait evolution, a discussion of which is beyond the scope of this paper.

A range of later works that considered the evolution of traits in co-evolving populations also cites this paper, and while it is often not the central paper considered, it is touched upon in a number of different approaches and scenarios. These include Mougi and Iwasa [118] which investigates when such oscillatory evolutionary behaviour will and will not occur, and Lion and van Baalen [104] which considers the influence of spatial factors in trait evolution. 


\subsection{Superparasitism of Hosts by Parasitoids}

Many wasp species are parasitoids which lay their eggs within the larvae of other species, eventually leading to the host's death. A larva can be parasitized by more than one egg [61], although only one offspring will survive; this is called superparasitism. Commonly females leave a pheromone mark on a host that they have parasitized and so can distinguish hosts that are unparasitized, parasitized by themselves or parasitized by conspecifics [96].

This behaviour was modelled in Haccou et al. [65], who considered a scenario with two parasitizing wasps $F_{v}$ and $F_{w}$ at a patch of host larvae. The first female enters a patch of non-parasitized hosts, the second arriving at a random time later. Each host can be in one of the five states: unparasitized, or parasitized by the first only, by the second only, by both (with the offspring of the first the survivor) or by both (with the offspring of the second the survivor). Females can distinguish the states, except the fourth from the fifth. They assumed that females began by parasitizing only unparasitized larvae (a non-superparasitizing female), perhaps switching at some time to both parasitizing and superparasitizing.

Non-superparasitizing females encounter (and parasitize) hosts at rate $\lambda$. Superparasitizing females encounter hosts at rate $\mu<\lambda$ and superparasitize if a host contains at least one egg of the other individual; this latest egg is the one to yield an offspring with probability $\sigma$.

The state of the patch is denoted by the proportions in the different cases $u$ (state 1, unparasitized hosts), $v$ (states 2 and 4, first female's offspring survives) and $w$ (states 3 and 5 , second female's offspring survives). The female strategy is a choice of leave, parasitize or superparasitize for every potential scenario, as either first or second female. The payoff to $F_{v}\left(F_{w}\right)$ is the derivative of $v(w)$ with respect to $t$. Different trajectories within the region $(u, v, w)$ occur for different strategies. For example when there is only a single superparasitizing female $F_{w}$ present, the trajectory follows

$$
\frac{\mathrm{d} u}{\mathrm{~d} t}=-\mu u, \frac{\mathrm{d} w}{\mathrm{~d} t}=\mu u+\sigma \mu v, \frac{\mathrm{d} v}{\mathrm{~d} t}=-\sigma \mu v .
$$

There is also a background environmental gain rate $\gamma$. At any point, the two females choose a strategy based upon how long they should wait, and when one leaves, the reward to the other will suddenly increase. This is equivalent to receiving a reward at that point, and Haccou et al. [65] thus analysed $F_{v}$ and $F_{w}$ as playing in a generalized war of attrition (see Sect. 2 and [9]).

The asymmetry of this model means that the first female to arrive effectively finds a better quality patch than the second, since there is a patch of completely unparasitized hosts. This led to the prediction that the first female would spend longer in the patch than the second. This prediction was empirically tested in Le Lann et al. [94] who conducted experiments with an aphid parasitoid Aphidius ervi Haliday in a patch of larvae from a grain aphid species Sitobion avenae Fabricius.

Three different scenarios were considered: (a) simultaneous patch exploitation with different arrival times, (b) exploitation of an unexploited patch by a single female, (c) exploitation of a previously exploited patch by a single female. The results found in this paper were entirely consistent with predictions made in Haccou et al. [65]. In particular, in patches where two individuals were present at the same time, the first arriving individual stayed for a longer time; indeed, it was usually the last to leave. 


\subsection{Sperm Competition}

Males can compete with each other directly for mates through fights, as represented by the Hawk-Dove game. However, they can also compete indirectly, and one key way they do this is through sperm competition. A series of sperm competition models was developed by Parker and colleagues (see e.g. [4,5,123]). An interesting alternative was developed by Chris and colleagues in Fryer et al. [58,59], which we describe below.

Consider a population consisting of a proportion $f$ females and $m=1-f$ males, which participates in two rounds of mating. Males will try to mate in each round, females mate only if receptive, and they may be receptive in either, neither or both rounds (in each round every receptive female will mate). $f_{i j}$ and $m_{i j}$ denote the proportion of males and females, respectively, who mate $i$ times in round 1 and $j$ times in round $2(i, j$ taking values 0 or 1$)$. The amount of sperm needed to guarantee fertilization is $\epsilon_{0}$. If two males' sperm compete for fertilization, any given sperm from the round 2 male is $r$ times more likely to fertilize the female than any given sperm from the round 1 male. The aim was to find the best male strategy $s$ of what proportion of sperm to invest in round 1 .

Setting $f_{1}=f_{10}+f_{11}$, the payoff to an $s^{*}$ player in a population of $s$ players was given by

$$
\begin{aligned}
E\left[s^{*}, s\right]= & \frac{f_{10}}{m} \min \left(\frac{s^{*}}{\epsilon_{0}}, 1\right)+\frac{f_{01}}{m} \frac{m-f_{1}}{m}+\frac{f_{01}}{m} \frac{f_{1}}{m} \min \left(\frac{1-s^{*}}{\epsilon_{0}}, 1\right) \\
& +\frac{f_{11}}{m}\left[\frac{m-f_{1}}{m}\left(\frac{s^{*}}{s^{*}+r}+\frac{r}{s+r}\right)+\frac{f_{1}}{m}\left(\frac{s^{*}}{s^{*}+r(1-s)}+\frac{r\left(1-s^{*}\right)}{s+r\left(1-s^{*}\right)}\right)\right] .
\end{aligned}
$$

They found that, except for non-generic cases, there is always a unique pure ESS value of $s$. This value was in general at least $1 / 2$ but could be any value up to 1 . The value of $1 / 2$ occurred principally when every male mates in the first round, i.e. $m=f_{1}$. The larger $m / f_{1}$, the larger $s$, and it could be that $s=1$ so all sperm is used in the first round (this occurred more commonly when $\epsilon_{0}$ was relatively large).

They also considered developments of this model, either where males could replenish some sperm between rounds or the females could oviposit some eggs between rounds. In each case, as expected, the ESS value of $s$ increases as a result.

The model of Fryer et al. [59] extended the above analysis to consider a guarding strategy, where instead of attempting to mate in the second round, a male could guard the female that he had mated with in the first round. If another male then attempted to mate with the female, this mating was prevented with probability $\phi$. They again found a unique ESS for each case, where this could either be monomorphic guarding, a monomorphic population of non-guarders with a single $s$ strategy (as in the original model where guarding was not allowed) or much more rarely a polymorphic mixture of guarding and a range of non-guarding strategies. An interesting recent model [160] made similar predictions to the above and was fitted with some success to data for three species.

\section{Games on Static Graphs}

Strategic interactions often occur in spatial situations where individuals only interact with their neighbours. This results in games on graphs. The types of games on graphs Chris studied can be separated into two categories. Firstly, the systems where the network remains static, 
and the strategies of individuals change over time. Secondly, Chris also considered games within which the network itself evolves as a result of the result of the strategic interactions of the nodes within.

We shall start by discussing systems where the network remains static, and the strategies employed by the nodes change over time. Many such models are reviewed in Szabo and Fath [156], although we will focus on the models inspired by Chris' research.

\subsection{Best Response Games}

In Southwell and Cannings [151], the state evolves over discrete-time steps, so that at each time step, each vertex simultaneously updates to the best response to their neighbours' strategies.

A best response game on a graph is specified by an undirected graph $G=(V, E)$, with a vertex set $V$ and an edge set $E$ and an $n$ by $n$ payoff matrix $M$. Vertices of the graph represent players. A state $s$ of the system, at time step $t$, is where each vertex $v$ of $G$ employs some pure strategy $s(v)$ in $\{1, \ldots, n\}$. The model update rules are such that, in the state $s^{\prime}$ at time step $t+1$, a vertex $v$ will employ the pure strategy $i$ in $\{1, \ldots, n\}$ which would have maximized their total payoff $\sum_{u:\{u, v\} \in E} M_{i, s(u)}$ against the strategy configuration their neighbours used at time step $t$. In other words, each player simultaneously updates to what they should have chosen against their current surroundings. Best response games of a similar nature were considered earlier in Berninghaus and Schwalbe [6,7]; however, the study by Southwell and Cannings [151] was the first work to examine the possible dynamics of games with more than two strategies. In Southwell and Cannings [151] a geometric method was used to enumerate the different best response games on various graph structures like the complete graph and the circle. The best response for a particular vertex just depends upon the fraction of its neighbours which are using the different pure strategies. This information can be represented as a point in the $n-1$-dimensional strategy simplex using barycentric coordinates. The type of dynamics of a game just depends upon how such discrete points are partitioned into different best response regions (i.e. regions of the strategy simplex which have different pure best responses). By considering the different ways to partition the strategy simplex into convex best response regions with respect to the discrete points (that represent local strategy configurations) Southwell and Cannings [151] were able to enumerate the best response games on simple graph structures like circles and complete graphs. A classification of the dynamics of two-strategy best response games on circle graphs was also given. When there are more than two strategies, best response games on the circle can have complex dynamics. For example, there is a three-strategy game which (when played on the infinite circle/line) corresponds to the chaotic cellular automata rule 90 (see [91,169]). Similarly complex dynamics have been observed in other types of games upon graphs [120].

\subsection{Majority/Minority Games}

Closer study of particular two-strategy best response games on graphs (and generalizations thereof) was carried out by Chris in Cannings [37]. In a majority game a player adopts the same strategy as the majority of its neighbours. The dual idea is the minority game, where a player adopts the same strategy as the minority of its neighbours. In Cannings [37] a combination of simulations and combinatorial methods was used to study such games on complete networks, hypercubes and a certain class of cubic networks. The different attractors of these dynamical systems, and their basins of attraction, were found in many cases. An 
interesting duality between the dynamics of minority and majority games was also noted. Further work along these lines was done in Haslegrave and Cannings [75] where it is shown that more interesting dynamics can take place when the network holds a single vertex that updates its strategy via different criteria.

The majority game can be thought of as a game on a graph, where matching a neighbour's strategy provides unit payoff, whereas not matching its strategy provides zero payoff. A generalization of this is where each vertex $v$ gets a payoff for using a strategy $i$ in $\{1, \ldots, n\}$ that is given by some (player, and strategy-specific) function of the number of neighbours using the same strategy. For such systems, Cannings and Cannings [40] settled the conjecture about the existence of a pure Nash equilibrium, negatively, by demonstrating the existence of such generalized coordination games with three players and three strategies which do not have pure Nash equilibria. This aided wider efforts to classify the game-theoretic properties of generalized network congestion games. These generalized congestion games have found numerous applications, especially for resource sharing in wireless networks $[152,154,159]$. Asynchronous updating is typically assumed in these systems, but results about Nash equilibria are useful irrespective of such details.

\section{Dynamic Network Models}

In addition to studying these games on static graphs, Chris also considered models on dynamic graphs. These dynamic models often yielded complex outcomes, even before considering any explicit game-theoretic element involving finding optimal strategic choices.

\subsection{Games with Reproducing Vertices}

We shall start by discussing such games where the vertices on a graph reproduce and die based on their success levels in local interactions (see [147,150]). As with the best response games, focus was on the deterministic case where the condition of each vertex is updated simultaneously. This allowed the usage of combinatorial methods to gain significant insights.

Such games on growing graphs are specified by an $n$ by $n$ payoff matrix, and a number called the fitness threshold. A system state is an undirected graph, where each vertex is associated with a pure strategy. The system is updated over discrete-time steps. A given update consists of two stages, the reproduction stage and then the killing stage. In the reproduction stage, each vertex simultaneously produces an offspring vertex, with the same strategy as the parent. There are eight possible models for how the connections of the offspring depends upon the parent, as we discuss below. For example, in the $m=1$ model the offspring just inherit their parent's neighbourhood (i.e. the offspring are born connected to the same individuals as the parent is connected to). After this reproduction stage, the second part of the update (the killing stage) consists of destroying all vertices that have a total payoff (added up, by playing a game with each neighbour, using their allocated strategies) that is below the fitness threshold.

Each of the eight different reproduction models is specified by three binary numbers $r_{0}, r_{1}, r_{2}$ in $\{0,1\}$. The parents always retain their pre-existing connections, but the offspring's connections are specified as follows:

- If $r_{0}=1$, then the offspring are connected to their parent's neighbours. Otherwise they are not.

- If $r_{1}=1$, then the offspring are connected to their parent. Otherwise they are not. 
Fig. 1 The vertex reproduction model of Southwell and Cannings [147]. In the version where reproduction occurs under the $m=1$ model and vertices with more than $Q=2$ links are killed, a single-edge self-replicates after two updates

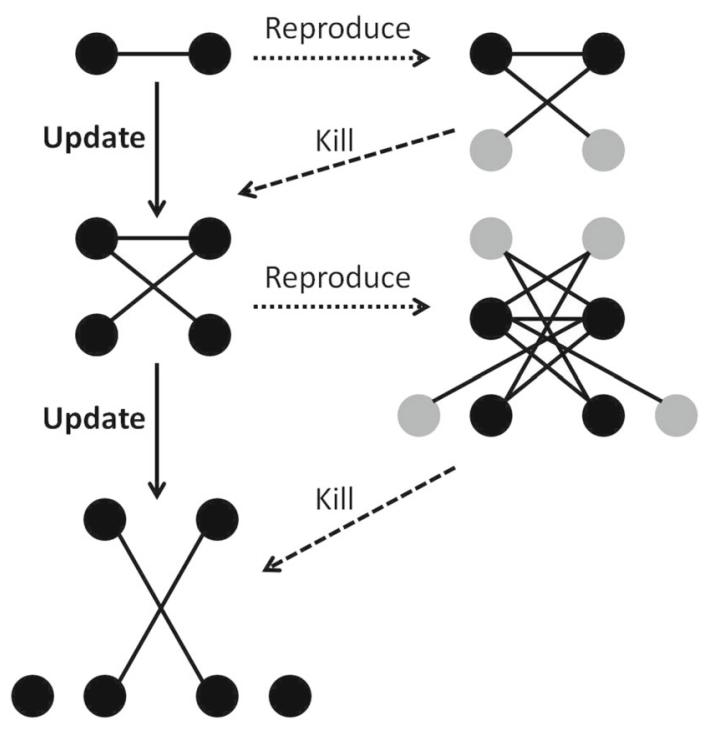

- If $r_{2}=1$, then the offspring of connected parents are connected to one another. Otherwise they are not.

The model specified by the binary string $r_{0}, r_{1}, r_{2}$ is called model number $m=r_{0} 2^{0}+r_{1} 2^{1}+$ $r_{2} 2^{2}$. In models with $m=0,2,4,5,6$ and 7 it is possible to gain significant understanding of the dynamics of general games $[147,150]$. However, extremely complex and interesting behaviour can be produced in the $m=1$ and $m=3$ models.

One case that was studied in detail $[147,150]$ is where there is just one strategy, which scores a payoff of -1 against itself, and the fitness threshold is $-Q$. In this case an individual survives the killing stage if and only if it has a degree less than $Q$, and so these systems correspond to a kind of degree capped reproduction where individuals reproduce, but die from overcrowding once they get more than $Q$ neighbours. When reproduction takes place according to the $m=1$ or $m=3$ models, these systems can generate complex self-replicating structures.

Figure 1 shows a simple example of a self-replicating structure under the $m=1$ model with degree cap $Q=2$. When the degree cap $Q$ is larger, much more interesting dynamics can occur, because the graph can grow larger before the highly connected vertices die. In many cases simple initial graph structures, like two vertices connected by a single edge, evolve to generate large collections of different connected components, which grow, change and self-replicate. For $Q=8$ the degree capped reproduction model (using reproduction method $m=1$ ) eventually produces 723 different self-replicating structures. More structures are produced for higher-degree cap values, and there are many unanswered questions about these systems. In particular, it is unknown whether systems with a finite degree cap $Q$ can generate connected components of arbitrary size or generate arbitrarily large numbers of non-isomorphic connected components. Studying these questions may give insight into how complex self-replicating structures arise from simple biologically inspired rules. Other types of model where the network structure changes as a result of strategic interaction were considered in Richter $[139,140]$. Also, the remarkably complex dynamics observed in Southwell and Cannings [147] inspired the further search for simple network evolution rules inducing complexity [153]. 


\subsection{Further Models with Reproducing Vertices}

In order to understand the basic network growth processes introduced in Southwell and Cannings [147], a study of the network dynamics under pure reproduction (i.e. with no gamebased death) was considered in Southwell and Cannings [148]. In this case many aspects of the dynamics of the degree distribution, chromatic number, diameter, adjacency matrix, etc., can be understood. In most cases the growth dynamics are quite simple and can be expressed by applying graph products. However, in model $m=3$, where each vertex is born connected just to its parent and its parent's neighbours, a complicated degree distribution arises, which was studied more closely in Jordan and Southwell [84]. In addition to studying the dynamics under pure reproduction, there has also been study of the systems where vertices die after they have survived more than a certain number of time steps [149]. The resulting systems could be considered to be a type of graphical generalization of the Leslie population model [97], where not only the number of individuals of different ages is modelled, but also the network structure connecting them. In Jordan [83] a generalization of the reproducing graph model was considered where links are formed at random.

\subsection{Link Formation Models}

A different type of model of network evolution was considered in Broom and Cannings [19], within which individuals form or break links at random, according to their degree. This forms an interesting model of sociability and behavioural response to epidemics. The basic idea is that each vertex has a pre-allocated minimum and maximum allowed degree. The system evolves via a Markov chain so that, at any given time, a vertex is chosen at random. If the vertex has more links than its maximum allowed degree, then one of its links is deleted randomly. If the vertex has less links than its minimum allowed degree, then a link is formed to a randomly selected unconnected individual. Otherwise no action is taken. Analysis revealed that these systems evolve to a closed class of structures, the minimal set, which Broom and Cannings [19] shows how to find the stationary distribution of, focusing on the special case where the maximum and minimum degrees are the same, i.e. each individual has a unique target.

Later work [20,21,39] explored the nature of the minimal set, classifying the vertices into four categories depending upon whether they always achieved their target, were never over target, never under target or could be under or over target. Interestingly, some vertices could be always on target even for a continuously changing process. They also investigated gametheoretic generalizations of this model, where formation and breaking of links also depend upon strategies employed by the vertices. In Broom and Cannings [21] in particular we can see that such games are inherently very complex, even for small populations.

\section{Discussion}

As we have described in this article Chris Cannings, together with varied collaborators, played an important part in the development of the theory of evolutionary games, including in the early stages of the development of the theory by John Maynard Smith. We have seen how his earliest work on evolutionary games helped establish the mathematical results on which the theory was based including the Bishop-Cannings theorem, which is a central result in the theory and is thus applied widely. It was particularly useful in a later theme of Chris' work, 
the investigation of patterns of ESSs, which considered matrix games and the possibilities and restrictions on what ESSs could occur in detail.

One of the earliest models developed was the war of attrition, and much of the theory of these games is due to Chris and collaborators, including the development of the generalized war of attrition. He also introduced a multiplayer version, which was a forerunner of his more general theory of multiplayer games, including games with structures such as knockout contests. Chris considered different kinds of structured games in later work, including games on static structures (as opposed to well-mixed populations) and also networks that were dynamically changing.

As well as developing the fundamental mathematical theory, Chris also worked on a range of models of real biological situations, for example on wasp superparasitism, which included an application of the generalized war of attrition which he had developed, and predator-prey co-evolution which used an early version of the method of adaptive dynamics.

His work has been influential in a number of areas which we have discussed in this paper. His early work on the war of attrition and on matrix games, and in particular the BishopCannings theorem, has become a fundamental part of the theory of evolutionary games. His work contributed near the start of John Maynard Smith's project and is therefore part of the foundation of evolutionary game theory. He was also one of the only researchers to try to span the theories of evolutionary games and population genetics, and this is still an area ripe for development. As he is the originator of the theory of patterns of ESSs and of multiplayer evolutionary games, modern work in both of these areas rests strongly on his early work. His innovative dynamic network models are also part of one of the most important areas of modern evolutionary theory.

The greatest tribute that can be paid to Chris, and the other early pioneers of evolutionary games, is that the work of a small number has developed into an ever-growing and complex field of enthusiastic young (and not so young) researchers who have built their models based upon the foundations they have laid. Chris was particularly noted for his enthusiasm for research, his wide range of interests and his mathematical intuition, combined with a deep knowledge. He was still producing new research until shortly before he died.

Acknowledgements This work was supported by funding from the European Union's Horizon 2020 research and innovation programme under the Marie Sklodowska-Curie Grant Agreement No. 690817. We would also like to thank all of the funders of Chris' work during his career as well as all of his research collaborators.

Open Access This article is licensed under a Creative Commons Attribution 4.0 International License, which permits use, sharing, adaptation, distribution and reproduction in any medium or format, as long as you give appropriate credit to the original author(s) and the source, provide a link to the Creative Commons licence, and indicate if changes were made. The images or other third party material in this article are included in the article's Creative Commons licence, unless indicated otherwise in a credit line to the material. If material is not included in the article's Creative Commons licence and your intended use is not permitted by statutory regulation or exceeds the permitted use, you will need to obtain permission directly from the copyright holder. To view a copy of this licence, visit http://creativecommons.org/licenses/by/4.0/.

\section{References}

1. Abakuks A (1980) Conditions for evolutionarily stable strategies. J Appl Probab 17:559-562

2. Apaloo J (1997) Revisiting strategic models of evolution: the concept of neighborhood invader strategies. Theor Popul Biol 52(1):52-71

3. Apaloo J, Brown J, Vincent T (2009) Evolutionary game theory: ESS, convergence stability, and NIS. Evol Ecol Res 11:489-515 
4. Ball M, Parker G (1997) Sperm competition games: inter- and intra-species results of a continuous external fertilization model. J Theor Biol 186(4):459-466

5. Ball M, Parker G (2007) Sperm competition games: the risk model can generate higher sperm allocation to virgin females. J Evol Biol 20(2):767-779

6. Berninghaus SK, Schwalbe U (1996a) Conventions, local interaction, and automata networks. J Evol Econ 6(3):297-312

7. Berninghaus SK, Schwalbe U (1996b) Evolution, interaction, and nash equilibria. J Econ Behav Organ 29(1):57-85

8. Bishop D, Cannings C (1976) Models of animal conflict. Adv Appl Probab 8:616-621

9. Bishop D, Cannings C (1978) A generalized war of attrition. J Theor Biol 70:85-124

10. Bishop D, Cannings C (1986) Ordinal conflicts with random rewards. J Theor Biol 122:225-230

11. Bishop D, Cannings C, Maynard Smith J (1978) The war of attrition with random rewards. J Theor Biol 74:377-388

12. Bomze I (1992) Detecting all evolutionarily stable strategies. J Optim Theory Appl 75(2):313-329

13. Bomze I (2002) Regularity versus degeneracy in dynamics, games, and optimization: a unified approach to different aspects. SIAM Rev 44(3):394-414

14. Bomze I, Schachinger W (2020) Constructing patterns of (many) ESSs under support size control. Dyn Games Appl

15. Bomze I, Schahcinger W, Ullrich R (2018) The complexity of simple models: a study of worst and typical hard cases for the standard quadratic optimization problem. Math Oper Res 43(2):651-674

16. Broom M (2000a) Bounds on the number of ESSs of a matrix game. Math Biosci 167:163-175

17. Broom M (2000b) Patterns of ESSs: the maximal pattern conjecture revisited. J Math Biol 40:406-412

18. Broom M, Cannings C (2002) Modelling dominance hierarchy formation as a multi-player game. J Theor Biol 219(3):397-413

19. Broom M, Cannings C (2013) A dynamic network population model with strategic link formation governed by individual preferences. J Theor Biol 335:160-168

20. Broom M, Cannings C (2015) Graphic deviation. Discret Math 338:701-711

21. Broom M, Cannings C (2017) Game theoretical modelling of a dynamically evolving network I: general target sequences. J Dyn Games 4:285-318

22. Broom M, Rychtár J (2012) A general framework for analyzing multiplayer games in networks using territorial interactions as a case study. J Theor Biol 302:70-80

23. Broom M, Cannings C, Vickers G (1993) On the number of local maxima of a constrained quadratic form. Proc R Soc Lond A 443:573-584

24. Broom M, Cannings C, Vickers G (1994) Sequential methods for generating patterns of ESS's. J Math Biol 32:597-615

25. Broom M, Cannings C, Vickers G (1996a) Choosing a nest site: contests and catalysts. Am Nat 147:11081114

26. Broom M, Cannings C, Vickers G (1996b) Patterns of ESS's. Adding pairs to an ESS. Math Biosci 136:21-34

27. Broom M, Cannings C, Vickers G (1997a) A sequential-arrivals model of territory acquisition. J Theor Biol 189:257-272

28. Broom M, Cannings C, Vickers G (1997b) Multi-player matrix games. Bull Math Biol 59(5):931-952

29. Broom M, Cannings C, Vickers G (2000a) A sequential-arrivals model of territory acquisition II. J Theor Biol 207:389-403

30. Broom M, Cannings C, Vickers G (2000b) Evolution in knockout conflicts: the fixed strategy case. Bull Math Biol 62(3):451-466

31. Broom M, Cannings C, Vickers G (2000c) Evolution in knockout contests: the variable strategy case. Selection 1(1):5-22

32. Broom M, Lafaye C, Pattni K, Rychtár J (2015) A study of the dynamics of multi-player games on small networks using territorial interactions. J Math Biol 71:1551-1574

33. Broom M, Pattni K, Rychtář J (2019) Generalized social dilemmas: the evolution of cooperation in populations with variable group size. Bull Math Biol. https://doi.org/10.1007/s11538-018-00545-1

34. Brown J, Vincent T (1992) Organization of predator-prey communities as an evolutionary game. Evolution 46:1269-1283

35. Bukowski M, Miękisz J (2004) Evolutionary and asymptotic stability in symmetric multi-player games. Int J Game Theory 33(1):41-54

36. Cannings C (1971) Natural selection at a multiallelic autosomal locus with multiple niches. J Genet 60:255-259

37. Cannings $C$ (2009) The majority game on regular and random networks. In: International conference on game theory for networks. IEEE, pp xii-xiii 
38. Cannings C (2015) Combinatorial aspects of Parker's model. Dyn Games Appl 5(2):263-274

39. Cannings C, Broom M (2020) Game theoretical modelling of a dynamically evolving network II: target sequences of score 1. J Dyn Games (accepted)

40. Cannings C, Cannings R (2013) Absence of pure nash equilibria in a class of co-ordination games. Stat Optim Inf Comput 1(1):1-7

41. Cannings C, Vickers G (1988) Patterns of ESS's 2. J Theor Biol 132:409-420

42. Cannings C, Vickers G (1990) Patterns and invasions of evolutionarily stable strategies. J Appl Math Comput 32:227-253

43. Cannings C, Vickers G (1991) The genealogy of patterns of ESS's. In: Basawa IV, Taylor RL (eds) Selected proceedings of the sheffield symposium on applied. Institute of Mathematical Statistics, Hayward, pp 193-204

44. Cannings C, Tyrer J, Vickers G (1993) Routes to polymorphism. J Theor Biol 165(2):213-223

45. Chappell J, Iqbal A, Abbott D (2012) N-player quantum games in an EPR setting. PLoS ONE 7(5):e36404

46. Chase I, Tovey C, Spangler-Martin D, Manfredonia W (2002) Individual differences versus social dynamics in the formation of animal dominance hierarchies. Proc Nat Acad Sci 99(8):5744-5749

47. Cressman R, Hines W (1984) Evolutionarily stable strategies of diploid populations with semi-dominant inheritance patterns. J Appl Probab 21:1-9

48. Dugatkin L (1997) Cooperation among animals: an evolutionary perspective. Oxford University Press, USA

49. Dugatkin L, Dugatkin A (2007) Extrinsic effects, estimating opponents RHP, and the structure of dominance hierarchies. Biol Lett 3(6):614-616

50. Duong M, Han T (2016) Analysis of the expected density of internal equilibria in random evolutionary multi-player multi-strategy games. J Math Biol 73(6):1727-1760

51. Duong M, Tran H (2018) On the fundamental solution and a variational formulation of a degenerate diffusion of Kolmogorov type. Discret Contin Dyn Syst Ser A 38(7):3407-3438

52. Duong M, Tran H, Han T (2019) On the distribution of the number of internal equilibria in random evolutionary games. J Math Biol 78:331-371

53. Eisert J, Wilkens M, Lewenstein M (1999) Quantum games and quantum strategies. Phys Rev Lett 83(15):3077-3080

54. Eshel I (1983) Evolutionary and continuous stability. J Theor Biol 103(1):99-111

55. Eshel I, Sansone E (2003) Evolutionary and dynamic stability in continuous population games. J Math Biol 46:445-459

56. Fishman M (2016) Polymorphic evolutionary games. J Theor Biol 398:130-135

57. Fishman M (2018) Animal conflicts in diploid populations with sexual reproduction. J Theor Biol 462:475-478

58. Fryer T, Cannings C, Vickers G (1999a) Sperm competition I: basic model, ESS and dynamics. J Theor Biol 196(1):81-100

59. Fryer T, Cannings C, Vickers G (1999b) Sperm competition II: post-copulatory guarding. J Theor Biol 197(3):343-360

60. Geritz S, Kisdi E, Meszéna G, Metz J (1998) Evolutionary singular strategies and the adaptive growth and branching of the evolutionary tree. Evol Ecol 12:35-57

61. Godfray H (1994) Parasitoids: behavioral and evolutionary ecology. Princeton University Press, Princeton

62. Gokhale C, Traulsen A (2010) Evolutionary games in the multiverse. Proc Nat Acad Sci 107(12):55005504

63. Gokhale C, Traulsen A (2011) Strategy abundance in evolutionary many-player games with multiple strategies. J Theor Biol 283(1):180-191

64. Gokhale C, Traulsen A (2014) Evolutionary multiplayer games. Dyn Games Appl 4(4):468-488

65. Haccou P, Glaizot O, Cannings C (2003) Patch leaving strategies and superparasitism: an asymmetric generalized war of attrition. J Theor Biol 225(1):77-89

66. Haigh J (1975) Game theory and evolution. Adv Appl Probab 7:8-11

67. Haigh J (1988) The distribution of evolutionarily stable strategies. J Appl Probab 25:233-246

68. Haigh J (1989) How large is the support of an ESS? J Appl Probab 26:164-170

69. Haigh J, Cannings C (1989) The n-person war of attrition. Acta Appl Math 14(1):59-74

70. Hamilton W (1967) Extraordinary sex ratios. Science 156:477-488

71. Hammerstein P (1981) The role of asymmetries in animal contests. Anim Behav 29(1):193-205

72. Hammerstein P, Parker G (1982) The asymmetric war of attrition. J Theor Biol 96:647-682

73. Han T, Traulsen A, Gokhale C (2012) On equilibrium properties of evolutionary multi-player games with random payoff matrices. Theor Popul Biol 81:264-272 
74. Hao Y, Wu Z (2018) Computation of sparse and dense equilibrium strategies of evolutionary games. Games 9:46-60

75. Haslegrave J, Cannings C (2017) Majority dynamics with one nonconformist. Discret Appl Math 219:3239

76. Helgesson P, Wennberg B (2015) The n-player war of attrition in the limit of infinitely many players. Dyn Games Appl 5:65-93

77. Hines WGS, Bishop DT (1984) On the local stability of an evolutionarily stable strategy in a diploid population. J Appl Probab 21(2):215-224

78. Hofbauer J, Sigmund K (1998) Evolutionary games and population dynamics. Cambridge University Press, Cambridge

79. Hofbauer J, Schuster P, Sigmund K (1979) A note on evolutionary stable strategies and game dynamics. J Theor Biol 81(3):609-612

80. Iqbal A, Toor A (2001) Entanglement and dynamic stability of Nash equilibria in a symmetric quantum game. Phys Lett Sect A Gen Atomic Solid State Phys 286(4):245-250

81. Iqbal A, Cheon T, Abbott D (2008) Probabilistic analysis of three-player symmetric quantum games played using the Einstein-Podolsky-Rosen-Bohm setting. Phys Lett Sect A Gen Atomic Solid State Phys 372(44):6564-6577

82. Iqbal I, Cheon T (2008) Evolutionary stability in quantum games. In: Abbott D, Davies PCW, Pati AK (eds)Quantum aspects of life, foreword by Sir Roger Penrose. Imperial College Press. ISBN 978-184816-267-9

83. Jordan J (2011) Randomised reproducing graphs. Electron J Probab 16:1549-1562

84. Jordan J, Southwell R (2010) Further properties of reproducing graphs. Appl Math 1(05):344

85. Kamiski D, Miekisz J, Zaborowski M (2005) Stochastic stability in three-player games. Bull Math Biol 67(6):1195-1205

86. Kimura M (1967) On the evolutionary adjustment of spontaneous mutation rates. Genet Res 9:23-34

87. Kingman J (1961) A mathematical problem in population genetics. Proc Camb Philos Soc 57:574-582

88. Kisdi E, Geritz S (1999) Adaptive dynamics in allele space: evolution of genetic polymorphism by small mutations in a heterogeneous environment. Evolution 53:993-1008

89. Kura K, Broom M, Kandler A (2015) Modelling dominance hierarchies under winner and loser effects. Bull Math Biol 77:927-952

90. Kura K, Broom M, Kandler A (2016) A game-theoretical winner and loser model of dominance hierarchy formation. Bull Math Biol 78:1259-90

91. Kurka P (2003) Topological and symbolic dynamics, vol 11. SMF, Marseille

92. Kuzmics C, Rodenburger D (2019) A case of evolutionarily stable attainable equilibrium in the laboratory. Econ Theory. https://doi.org/10.1007/s00199-019-01224-5

93. Le Galliard J, Ferriere R, Dieckmann U (2003) The adaptive dynamics of altruism in spatially heterogeneous populations. Evolution 57(1):1-17

94. Le Lann C, Outreman Y, van Alphen J, van Baaren J (2011) First in, last out: asymmetric competition influences patch exploitation of a parasitoid. Behav Ecol 22(1):101-107

95. Leinster T, Meckes M (2016) Maximizing diversity in biology and beyond. Entropy 18(3):88

96. van Lenteren J (1981) Host discrimination by parasitoids. In: Nordlund DA, Jones RL, Lewis WL (eds) Semiochemicals: their role in pest control. Wiley, New York, pp 153-180

97. Leslie P (1945) On the use of matrices in certain population mathematics. Biometrika 1:183-212

98. Lessard S (1990) Evolutionary stability: one concept, several meanings. Theor Popul Biol 37:159-170

99. Li A, Wu B, Wang L (2014) Cooperation with both synergistic and local interactions can be worse than each alone. Sci Rep 4:5536

100. Li A, Broom M, DuJ Wang L (2016) Evolutionary dynamics of general group interactions in structured populations. Phys Rev E 93:022407

101. Lieberman E, Hauert C, Nowak M (2005) Evolutionary dynamics on graphs. Nature 433(7023):312-316

102. Lindquist W, Chase I (2009) Data-based analysis of winner-loser models of hierarchy formation in animals. Bull Math Biol 71(3):556-584

103. Lindquist W, Chase I (2016) The fragility of individual-based explanations of social hierarchies: a test using animal pecking orders. PLoS ONE 11(7):e0158900

104. Lion S, van Baalen M (2008) Self-structuring in spatial evolutionary ecology. Ecol Lett 11(3):277-295

105. Lotka A (1925) Elements of physical biology. Williams \& Wilkins, Philadelphia

106. Marrow P, Cannings C (1993) Evolutionary instability in predator-prey systems. J Theor Biol 160:135150

107. Marrow P, Law R, Cannings C (1992) The coevolution of predator-prey interactions: ESSs and red queen dynamics. In: Proceedings: biological sciences, pp 133-141 
108. Maynard Smith J (1974) The theory of games and the evolution of animal conflicts. J Theor Biol 47(1):209-221

109. Maynard Smith J, Price G (1973) The logic of animal conflict. Nature 246:15-18

110. McAvoy A, Hauert C (2015) Structure coefficients and strategy selection in multiplayer games. J Math Biol 72:203-238

111. Mesterton-Gibbons M, Dugatkin L (1995) Toward a theory of dominance hierarchies: effects of assessment, group size and variation in fighting ability. Behav Ecol 6(4):416-423

112. Mesterton-Gibbons M, Sherratt T (2014) Bourgeois versus anti-bourgeois: a model of infinite regress. Anim Behav 89:171-183

113. Mesterton-Gibbons M, Karabiyik T, Sherratt T (2014) The iterated Hawk-Dove game revisited: the effect of ownership uncertainty on bourgeois as a pure convention. Dyn Games Appl 4:407-431

114. Mesterton-Gibbons M, Karabiyik T, Sherratt T (2016) On the evolution of partial respect for ownership. Dyn Games Appl 6:359-395

115. Miekisz J (2004) Stochastic stability in spatial three-player games. Physica A 343:175-18

116. Miekisz J, Matuszak M, Poleszczuk J (2014) Stochastic stability in three-player games with time delays. Dyn Games Appl 4(4):489-498

117. Miekisz J, Bodnar M, Vardanyan R (2020) Three-player games with strategy-dependent time delays. Dyn Games Appl (accepted)

118. Mougi A, Iwasa Y (2010) Evolution towards oscillation or stability in a predator-prey system. Proc R Soc B Biol Sci 277:3163-3171

119. Nowak M (2006) Evolutionary dynamics, exploring the equations of life. Harvard University Press, Cambridge

120. Nowak MA, May RM (1992) Evolutionary games and spatial chaos. Nature 359(6398):826

121. Ohtsuki H, Hauert C, Lieberman E, Nowak M (2006) A simple rule for the evolution of cooperation on graphs and social networks. Nature 441(7092):502-505

122. Orlin Grabbe $\mathbf{J}$ (2005) An introduction to quantum game theory. arXiv arXiv:0004.192005

123. Parker G (1982) Why are there so many tiny sperm? Sperm competition and the maintenance of two sexes. J Theor Biol 96(2):281-294

124. Parker GA (1984) Evolutionarily stable strategies. In: Krebs JR, Davies NB (eds) Behavioural ecology: an evolutionary approach. Blackwell, Oxford, pp 30-61

125. Pattni K, Broom M, Rychtáŕ J (2017) Evolutionary dynamics and the evolution of multiplayer cooperation in a subdivided population. J Theor Biol 429:105-115

126. Pattni K, Broom M, Rychtár J (2018) Evolving multiplayer networks: modelling the evolution of cooperation in a mobile population. Discret Contin Dyn Syst B 23:1975-2004

127. Pelillo M, Rota Bulo S (2014) Clustering games. Stud Comput Intell 532:157-186

128. Pena J, Noldeke G (2016) Variability in group size and the evolution of collective action. J Theor Biol 389:72-82

129. Pena J, Noldeke G (2018) Group size effects in social evolution. J Theor Biol 457:211-220

130. Pena J, Rochat $Y$ (2012) Bipartite graphs as models of population structures in evolutionary multiplayer games. PLoS ONE 7(9):e44514

131. Pena J, Lehmann L, Noldeke G (2014) Gains from switching and evolutionary stability in multi-player matrix games. J Theor Biol 346:23-33

132. Pena J, Wu B, Arranz J, Traulsen A (2016a) Evolutionary games of multiplayer cooperation on graphs. PLoS Comput Biol 12(8):e1005059

133. Pena J, Wu B, Traulsen A (2016b) Ordering structured populations in multiplayer cooperation games. J R Soc Interface 13:20150881

134. Platkowski T (2004) Evolution of populations playing mixed multiplayer games. Math Comput Modell 39(9-10):981-989

135. Platkowski T (2016) Evolutionary coalitional games. Dyn Games Appl 6(3):396-408

136. Płatkowski T, Bujnowski P (2009) Cooperation in aspiration-based N-person prisoner's dilemmas. Phys Rev E 79(3):036103

137. Platkowski T, Stachowska-Pietka J (2005) ESSs in n-player mixed games. Appl Math Comput 167(1):592-606

138. Rapoport A (1985) Applications of game-theoretic concepts in biology. Bull Math Biol 47:161-192

139. Richter H (2016) Analyzing coevolutionary games with dynamic fitness landscapes. In: IEEE congress on evolutionary computation (CEC). IEEE, pp 609-616

140. Richter H (2017) Dynamic landscape models of coevolutionary games. BioSystems 153:26-44

141. Richter H (2019) Properties of network structures, structure coefficients, and benefit-to-cost ratios. Biosystems 180:88-100 
142. Rota Bulo S, Pelillo M (2009) A game-theoretic approach to hypergraph clustering. In: Proceedings of the 2009 conference on advances in neural information processing systems, vol 22, pp 1571-1579

143. Sandholm W (2010) Population games and evolutionary dynamics. MIT Press, Cambridge

144. Santos F, Santos M, Pacheco J (2008) Social diversity promotes the emergence of cooperation in public goods games. Nature 454:213-216

145. Selten R (1980) A note on evolutionarily stable strategies in asymmetric animal conflicts. J Theor Biol 84:93-101

146. Sherratt T, Mesterton-Gibbons M (2015) The evolution of respect for property. J Evol Biol 28:1185-1202

147. Southwell R, Cannings C (2009) Games on graphs that grow deterministically. In: International conference on game theory for networks. IEEE, pp 347-356

148. Southwell R, Cannings C (2010a) Some models of reproducing graphs: I pure reproduction. Appl Math 1(03):137

149. Southwell R, Cannings C (2010b) Some models of reproducing graphs: II age capped vertices. Appl Math 1(04):251

150. Southwell R, Cannings C (2010c) Some models of reproducing graphs: III game based reproduction. Appl Math 1(05):335

151. Southwell R, Cannings C (2013) Best response games on regular graphs. Appl Math 4:950-962

152. Southwell R, Chen Y, Huang J, Zhang Q (2012a) Convergence dynamics of graphical congestion games. In: International conference on game theory for networks. Springer, pp 31-46

153. Southwell R, Huang J, Cannings C (2012b) Complex networks from simple rewrite systems. arXiv preprint arXiv: 1205.0596

154. Southwell R, Chen X, Huang J (2014) Quality of service games for spectrum sharing. IEEE J Sel Areas Commun 32(3):589-600

155. Stanley R (2001) Enumerative combinatorics, vol 2. Cambridge University Press, Cambridge

156. Szabo G, Fath G (2007) Evolutionary games on graphs. Phys Rep 446(4-6):97-216

157. Tarnita C, Antal T, Ohtsuki H, Nowak M (2009) Evolutionary dynamics in set structured populations. Proc Nat Acad Sci 106(21):8601-8604

158. Taylor P, Jonker L (1978) Evolutionarily stable strategies and game dynamics. Math Biosci 40:145-156

159. Tekin C, Liu M, Southwell R, Huang J, Ahmad SHA (2012) Atomic congestion games on graphs and their applications in networking. IEEE/ACM Trans Netw 20(5):1541-1552

160. Turnell B, Shaw K, Reeve H (2018) Modeling strategic sperm allocation: tailoring the predictions to the species. Evolution 72(3):414-425

161. Vickers G, Cannings C (1987) On the definition of an evolutionarily stable strategy. J Theor Biol 129:349-353

162. Vickers G, Cannings C (1988a) On the number of stable equilibria in a one-locus, multi-allelic system. J Theor Biol 131(3):273-277

163. Vickers G, Cannings C (1988b) Patterns of ESS's 1. J Theor Biol 132:387-408

164. Vincent T, Brown J (1988) The evolution of ESS theory. Annu Rev Ecol Syst 19:423-443

165. Vincent T, Brown J (2005) Evolutionary game theory, natural selection and Darwinian dynamics. Cambridge University Press, Cambridge

166. Vincent TL, Brown JS (1984) Stability in an evolutionary game. Theor Popul Biol 26(3):408-427

167. Volterra V (1926) Variazioni e fluttuazioni del numero dindividui in specie animali conviventi. Mem Accad Sci Lincei 2:31-113

168. Wang N, Zhou W, Wu Z (2018) Equilibrium distributions of populations of biological species on networks of social sites. J Biol Dyn. https://doi.org/10.1080/1751375820181508762

169. Wolfram S (2002) A new kind of science, vol 5. Wolfram Media, Champaign

170. Wu B, Traulsen A, Gokhale C (2013) Dynamic properties of evolutionary multi-player games in finite populations. Games 4(2):182-199

171. Wu B, Arranz J, Du J, Zhou D, Traulsen A (2016) Evolving synergetic interactions. J R Soc Interface $13: 20160282$

Publisher's Note Springer Nature remains neutral with regard to jurisdictional claims in published maps and institutional affiliations. 